Th4.4 4:50pm - 5:10pm

\title{
3-D HOLOGRAPHIC DISKS
}

Hsin-Yu Sidney Li, Kevin Curtis, Yong Qiao, and Demetri Psaltis

Department of Electrical Engineering, CALTECH

Pasadena, CA 91125, tel: (818)356-4856

The 3-D holographic disk [1] (HD) is shown in Fig. 1. It consists of a photosensitive material shaped as a disk. Information is holographically stored throughout the volume of the thick disk using angle $(\theta)$ [2] or wavelength $(\lambda)$ multiplexing [3]. By tuning either the wavelength or the angle of the reference beam, multiple holograms of data blocks are superimposed at each location. The stored data is retrieved by using the disk/head motion to address a desired location and then selecting the angle or wavelength of the reconstructing beam to retrieve any one block of data. The system shown in Fig. 1 is an angle multiplexed system with an acoustooptic device used to to rapidly select the angle of illumination.

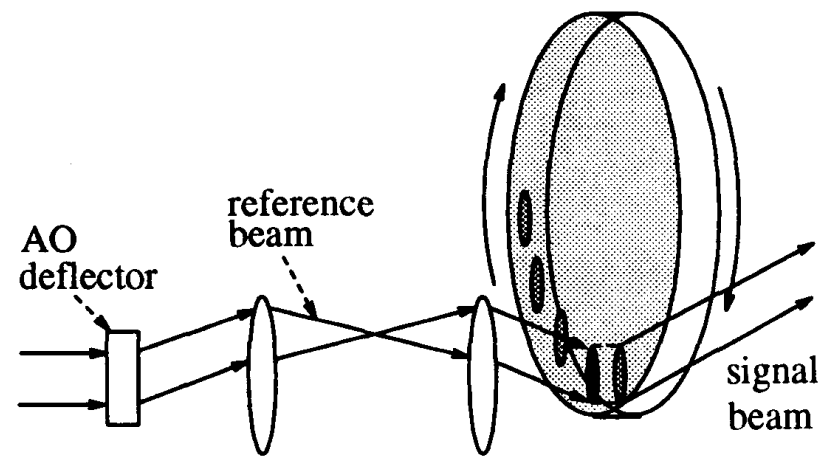

Fig. 1. 3-D holographic disk

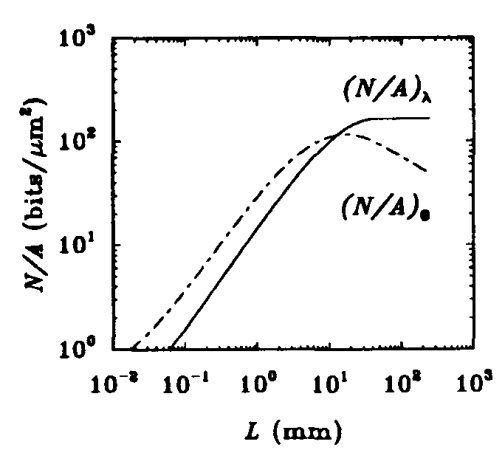

Fig. 2. Capacity vs. thickness

The advantage of HD's is large storage density. A conventional disk stores approximately 1 bit per $\mu \mathrm{m}^{2}$. In Fig. 2 we plot the maximum storage density obtained by an HD using $\theta$ or $\lambda$ multiplexing as a function of the disk thickness. The density is roughly 100 bits per micron squared in both cases for a thickness of approximately $1 \mathrm{~cm}$. Several parameters must be optimally selected to achieve the optimum density. In Fig. 2 each data block consists of $10^{3} \times 10^{3}$ bits and 1,800 superimposed holograms for $\lambda$ and 5,200 holograms for $\theta$ multiplexing. The density for $\theta$ and $\lambda$ multiplexing reaches a maximum in both cases for a thickness of about $1.5 \mathrm{~cm}$. As the thickness of the hologram increases, the $\theta$ and $\lambda$ selectivity increases, allowing us to superimpose more holograms. However, the area of each location increases because light is defocused as it propagates through the thick disk. This phenomenon explains the saturation for the $\lambda$ case. The recording area increases faster with disk thickness for $\theta$ multiplexing because the read-out beam is incident at an angle and it requires larger recording area.

The high storage density of the HD is achieved primarily by superimposing several thousand holograms in the same volume. This immediately raises the issue of the dynamic range of the material. Photorefractive crystals and photopolymers are the two materials we have used to fabricate HD's. In photorefractives up to 5,000 holograms have been superimposed in $1 \mathrm{~cm}$ thick crystals with raw probability of error less than $10^{-10}$ [4]. We have also demonstrated that photopolymers can also support the same density [5] except we have not been able so far to fabricate disks with thickness more than $300 \mu \mathrm{m}$. Photopolymers, are relatively inexpensive and easy to fabricate and they can be fixed for permanent (write-once) storage. Photorefractives are dynamic media and they are best suited for reprogrammable memories. However, nondestructive read-out and selective erasure are not normally possible. To overcome this problem we use a "dynamic holographic memory" [6] in which the disk contents are periodically read-out, amplified and re-recorded on the disk. Fig. 3a shown the reconstruction of 10 holograms superimposed at one location. Fig. $3 \mathrm{~b}$ shows the same data reconstructed after one of the data blocks (holograms) has been erased completely and the rest have been partially erased. In Fig. 3c the partially erased holograms have recovered their original strength through copying. Finally a new block of data is recorded in the blank location completing the read/erase/write/read cycle (Fig. 3d). 


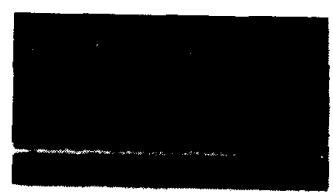

(a)

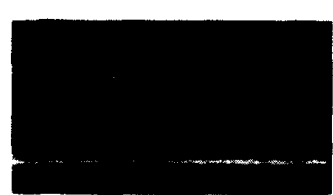

(b)

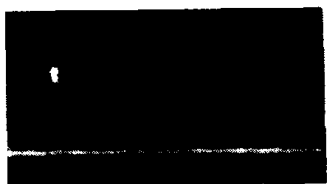

(c)

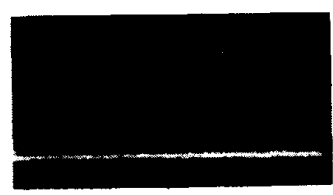

(d)

Fig. 3. Cycle of a dynamic holographic memory.

We fabricate either photorefractive or photopolymer based HD's. The photopolymer HD's are made with DuPont's HRF-150 or HRF-352 materials. We either use films provided by DuPont or we spin-coat material from a solution. Typically, the thickness of the spin-coated photopolymer disks is $\sim 200 \mu \mathrm{m}$ yielding a recording density of only $27 \mathrm{bits} / \mu \mathrm{m}^{2}$ with $\mathrm{F} / 1$ optics and $\lambda$ multiplexing. The calculation in Fig. 2 assumes $\mathrm{F} / 3$ lenses. Fig. 4 shows the readout from the photopolymer HD from 100 different locations on the disk. The uniformity of the recording is $49 \%$ over the disk surface. The photorefractive HD is fabricated with a c-cut iron-doped $\mathrm{LiNbO}_{3}$ wafer that is $5 \mathrm{~mm}$ thick and 1.5 inch in diameter. In one experiment, a short video was recorded by storing holograms at 20 different locations and storing 100 frames (holograms) at each location. Approximately $10^{8}$ bits were stored, which is a tiny fraction of the available space since from Fig. 2 the total storage capacity of this small disk is found to be approximately $10^{11}$ bits.
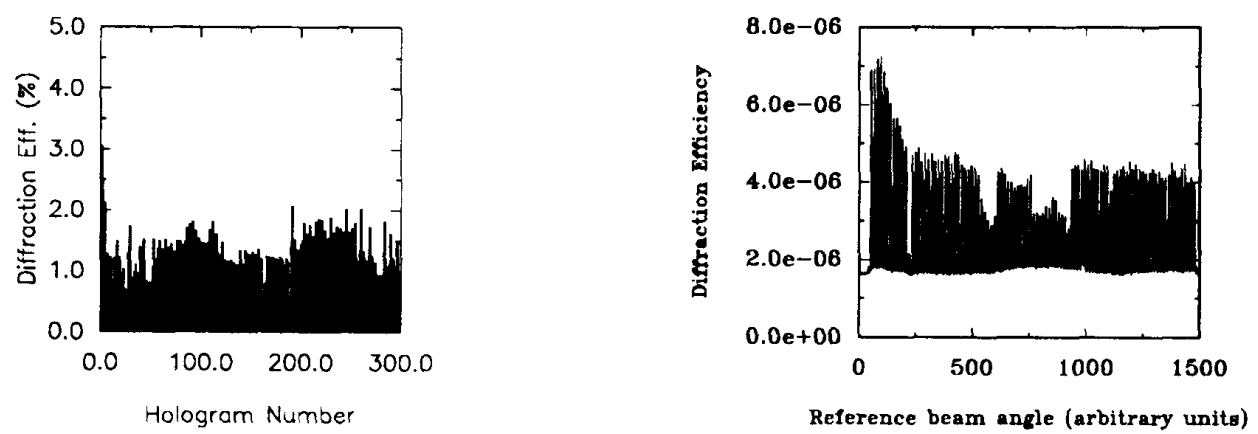

Fig. 4 Diffraction efficiency vs. hologram number for (a) photopolymer WORM disk, and (b) photorefractive read-write disk.

This work is supported by the Air Force Office of Scientific Research.

\section{References}

[1] Demetri Psaltis, "Parallel optical memories", Byte, 17(9), 179-182 (1992).

[2] D. L. Staebler, W. J. Burke, W. Phillips, and J. J. Amodei, "Multiple Storage and Erasure of Fixed Holograms in Fe-Doped LiNbO 3 ," Appl. Phys. Lett., 26(4), 182-184 (1975).

[3] G.A. Rakuljic, V. Leyva, and A. Yariv, "Optical-data Storage by using Orthogonal WavelengthMultiplexed Volume Holograms," Opt. Lett., 17(20), 1471-1473 (1992).

[4] F.H. Mok, "Applications of Holographic Storage in Lithium Niobate," (presented at OSA 1992 Annual Meeting) OSA 1992 Annual Meeting Technical Digest, Vol. 23, WE1, 102, Sept 1992.

[5] K. Curtis and D. Psaltis, "Recording of multiple holograms in photopolymer films," Appl. Opt., 31(35), 7425-7428 (1992).

[6] Y. Qiao and D. Psaltis "Sampled Dynamic Holographic Memory," Opt. Lett., 17(19), 1376-1378 (1992). 